Research Paper

\title{
Dual Delivery of NGF and bFGF Coacervater Ameliorates Diabetic Peripheral Neuropathy via Inhibiting Schwann Cells Apoptosis
}

Rui $\mathrm{Li}^{1 *}$, Jianfeng $\mathrm{Ma}^{1}{ }^{2 *}$, Yanqing $\mathrm{Wu}^{3 *}$, Matthew Nangle${ }^{2}$, Shuang Zou1 ${ }^{1}$, Yiyang $\mathrm{Li}^{1}$, Jiayu Yin ${ }^{1}$, Yingzheng $\mathrm{Zhao}^{1}$, Helin $\mathrm{Xu}^{1}$, Hongyu Zhang ${ }^{1}$, Xiaokun $\mathrm{Li}^{3}$, Qing song Ye ${ }^{1,2 凶}$, Jian Wang ${ }^{4 凶}$, Jian Xiao ${ }^{1 凶}$

1. WZMU-JCU Joint Research Group for Stem Cell and Tissue Engineering, Institute of Stem Cell and Tissue Engineering, School of Pharmacy, Wenzhou Medical University, Wenzhou 325035, China;

2. UQ-WMU Joint Research Group for Regenerative Medicine, Oral Health Centre, University of Queensland, Brisbane 4006, Australia;

3. The Institute of Life Sciences, Wenzhou University, Wenzhou 325035, China;

4. Department of Peripheral Neurosurgery, the First Affiliated Hospital Wenzhou Medical University, Wenzhou, Zhejiang 325035, China.

* These authors contributed equally to this work.

凹 Corresponding authors: Jian Xiao, xfxj2000@126.com; Jian Wang, jianwang0516@126.com; Qingsong Ye, qingsongye@hotmail.com

(c) Ivyspring International Publisher. This is an open access article distributed under the terms of the Creative Commons Attribution (CC BY-NC) license (https:// creativecommons.org/licenses/by-nc/4.0/). See http://ivyspring.com/terms for full terms and conditions.

Received: 2016.12.05; Accepted: 2017.03.09; Published: 2017.05.16

\begin{abstract}
Diabetic neuropathy is a kind of insidious complications that impairs neural and vascular function and ultimately leads to somatic and visceral denervation. Basic fibroblast growth factor (bFGF) and nerve growth factor (NGF) are important neurotrophic factors for stimulating angiogenesis and improving peripheral nerve function. Administrating a single factor has good therapeutic effect on diabetic peripheral neuropathy (DPN). However, the short half-life and rapid diffusion of growth factors under physiological conditions limits its clinical applications. Here, we used a biodegradable coacervate, composed of heparin and polycation, to dominate the combined release of bFGF and NGF in a steady fashion. We found this combined growth factors (GFs) coacervate, administered as a single injection, improved motor and sensory functions, restored morphometric structure and decreased apoptosis of Schwann cells in a rat model of prolonged DPN. Similarly the GFs coacervate, as compared with free bFGF and NGF combination, markedly reduced the apoptosis level of a rat Schwann cell line, RSC 96 cells in vitro. We also demonstrated that neuroprotective effects of the GFs coacervate in both rat DPN model and hyperglycemia-induced RSC 96 cell model is likely due to suppression of endocytoplasmic reticulum stress (ERS).
\end{abstract}

Key words: diabetic peripheral neuropathy (DPN); schwann cells (SCs); basic fibroblast growth factor (bFGF); nerve growth factor (NGF); coacervate.

\section{Introduction}

Diabetic peripheral neuropathy (DPN) as one kind of the most common and chronic complications of diabetes mellitus occur in approximately $50 \%$ of all diabetic patients $[1,2]$. It results in pain, paraesthesia, and decreased sensation, and can negatively influence quality of life [3]. Currently, there are no specific therapies to successfully prevent DPN except glycemic control [4]. Nevertheless, maintaining stable blood glucose is often difficult to achieve in many patients. Therefore, it needs to further explore the better treatment for DPN.

Recent evidence has indicated that endoplasmic reticulum stress (ERS) exerts an essential role in the onset and progression of DPN [5]. ERS is being increasingly considered as one of the main molecular mechanisms underlying DPN [6]. The endoplasmic reticulum (ER) is a sophisticated membrane system which exerts a vital role in the process of newly 
proteins synthesis and folding, and the storage of calcium. Pathological cellular stressors that disrupt ER homeostasis such as nutrient deficiency, oxidative stress, perturbation of calcium homeostasis, and increase of unsaturated fatty acids or cholesterol, can all induce ERS [7, 8]. As counter measures for ERS, cells carry out unfolding protein response (UPR) that includes: reducing the excessive folding of proteins, up-regulating the synthesis of related-chaperones and enzymes. These measures promote the ability of proteins to fold properly, and reinforce the self-repair ability of the ER [9, 10]. Effective new treatment methods for DPN must thus target the ERS-UPR pathway.

Basic fibroblast growth factor (bFGF), a neurotrophic factor, can play the part of a crucial regulator for neuroprotection, neurogenesis and angiogenesis from various insults [11]. Nerve growth factor (NGF), the first discovered neurotrophin, has been extensively shown to promote survival and maintain neuronal function [12]. Both factors subserve the maintenance and development of neurons and facilitate neural repair in the peripheral nervous system (PNS). It has been reported that diabetes significantly reduced the production and secretion of bFGF and NGF in sciatic nerve tissue, and exogenously administrated either of these two neurotrophic factors (NTFs) improved morphological and functional recovery [13-15]. However, a therapeutic strategy for DPN, based on a single growth factor (GF), may not be sufficient to provide robust neuroprotective effects because neural network comprises different neurons that requires different NTFs [16].

In a previous study, we developed an injectable coacervate, made of a polycation, poly (ethylene arginyl aspartate diglyceride) (PEAD) and GF-binding heparin, which easily bound a various of growth factors (GFs) and controlled its release in a steady way [17-19]. Here, we investigated whether use of bFGF and NGF in combination could prevent the progression or promote the recovery of diabetic neuropathy in streptozotocin (STZ)-induced diabetic rats. Since both of these two GFs are proteins, they are unstable and degrade rapidly in vivo $[20,21]$, thus it would be ideal to control co-delivery of these two GFs for promoting nerve repair.

In our current study, we had developed an injectable GFs coacervate with bFGF and NGF, and tried to reveal whether GFs coacervate exerts a better protective role on DPN. Additionally, we also explored the molecular mechanism underlying the protective role of GFs coacervate on DPN, aiming to provide the theoretical basis for treatment of DPN.

\section{Materials and Methods}

\section{Reagents and antibodies}

Recombinant human bFGF was purchased from Grost (Grost Biotechnology, Zhejiang, China). NGF, heparin and streptozotocin (STZ) were purchased from Sigma (Sigma-Aldrich, St. Louis, MO, USA). PEAD was a gift from University of Pittsburgh. Hematoxylin and eosin were purchased from Beycotime Biotechnology. NGF and bFGF enzyme linked immunosorbent assay (ELISA) kits were purchased from Elabscience Biotechnology. Dulbecco's modified Eagle's medium (DMEM) and fetal bovine serum (FBS) were purchased from Invitrogen (Carlsbad, CA, USA). Antibodies against GRP-78, ATF-6, ATF-4 and Caspase-12 were purchased from Abcam Biotechnology (Cambridge, MA, USA). Anti-CHOP and anti-XBP-1 were purchased from Santa Cruz Biotechnology (Santa Cruz, CA, USA). Anti-GAPDH was purchased from Bioworld Biotechnology.

\section{Growth Factor release assay}

Poly (ethylene argininylaspartate diglyceride) (PEAD) was synthesized as previously described [22]. PEAD, heparin, NGF and bFGF were seriatim dissolved in $0.9 \%$ normal saline to obtain $10 \mathrm{mg} \mathrm{mL}^{-1}$ solutions and sterilized using $0.22 \mu \mathrm{m}$ Millipore filter. The release assay in vitro $(\mathrm{n}=3)$ was performed using $1 \mu \mathrm{l}$ of NGF and $1 \mu \mathrm{l}$ of bFGF combined together, then mixed with $10 \mu \mathrm{l}$ of heparin followed lastly by the addition of $50 \mu \mathrm{l}$ of PEAD solution. On Day 1, 4, 7, 14, 21,28 and 35 , the coacervate was gently mixed and centrifuged at $12,000 \mathrm{~g}$ for $10 \mathrm{~min}$. Then, the supernatant was collected and stored, and $50 \mu \mathrm{l}$ of fresh saline was added to the pellet. According to the ELISA kit manufacturer's instructions, the collected supernatant was measured by ELISA to detect the amount of released growth factor.

\section{Induction of DPN and drug treatment}

Eight-week old male Wistar rats (200-220 g) were purchased from the Animal Center of the Chinese Academy of Sciences in Shanghai, China. The protocol for animal care and use was conformed to the Guide for the Care and Use of Laboratory Animals from the National Institutes of Health and was approved by the Animal Care and Use Committee of Wenzhou Medical University. Animals were maintained in an aseptic animal room at least 1 week before the experiment with a temperature of $20-24^{\circ} \mathrm{C}$ on a 12-h light/dark cycle and free access to food and water. Diabetes was induced by intraperitoneally injecting STZ with a dose of $65 \mathrm{mg} / \mathrm{kg}$ in phosphate-buffered saline (PBS). Control group 
received an equal volume of PBS. After $48 \mathrm{~h}$, rats were detected the blood glucose, and the concentrations $\geq 16.7 \mathrm{mmol} / \mathrm{L}$ were considered as diabetes [23, 24]. After 8 weeks, diabetic rats were randomly divided into three groups: STZ-induced diabetes, free GFs (NGF + bFGF) and GFs coacervate ([PEAD:heparin: $\mathrm{NGF}+\mathrm{bFGF}])$. Each groups contained eight rats. For the GFs coacervate group, $0.1 \mathrm{ml}$ saline solution $(60 \mu \mathrm{g}$ NGF, $60 \mu \mathrm{g}$ bFGF, $1.2 \mathrm{mg}$ heparin and $6 \mathrm{mg}$ PEAD) was injected into the right thigh and soleus muscles through a $1.0 \mathrm{ml}$ syringe only once. However, for the free GFs group, the dosages of NGF and bFGF were decided according to Mika's report [14], in which animals were administered intramuscular $20 \mu \mathrm{g}$ NGF and $20 \mu \mathrm{g}$ bFGF once daily for 3 consecutive days. STZ-induced diabetes group was administrated with the same volume of saline and control animals did not receive any treatment. After 30 days, the rats were anesthetized with $4 \%$ choral hydrate $\left(10 \mathrm{ml} \mathrm{kg}^{-1} \mathrm{IP}\right)$ and then perfused with $0.9 \% \mathrm{NaCl}$. The sciatic nerves from both sides were dissected out and harvested, and the pathology index was assessed.

\section{Hot Plate Test}

Hot plate test was to evaluate the sensory functional recovery of animals by measuring hind paw's licking and shaking. The test was conducted by two independent examiners who were blinded to remedy and to record sensory recovery on weekly bases after drug administration. Briefly, a hot plate at $55 \pm 1^{\circ} \mathrm{C}$ was prepared for the test, and the animals were positioned to stand with the operated hind paw on the hot plate. Thermal withdrawal reflex (TWRL) was measured by recording the time between placing in the hot plate and shaking or licking the paws. The cut-off time was set at $20 \mathrm{~s}$ to minimize skin injury. All tests were repeated for 4 times with a 5 min interval. If no hind paw withdraw was observed after $20 \mathrm{~s}$, the TWRL was considered as $20 \mathrm{~s}$.

\section{Walking track analysis}

Walking track analysis was carried out each week after treatment to assess motor functional recovery, and the sciatic function index (SFI) value was calculated using the method proposed by Bain et al [25]. The formula for calculation is as follows:

$$
\begin{gathered}
\mathrm{SFI}=-38.3 \times(\mathrm{EPL}-\mathrm{NPL}) / \mathrm{NPL}+109.5 \times(\mathrm{ETS}- \\
\mathrm{NTS}) / \mathrm{NTS}+13.3 \times(\mathrm{EIT}-\mathrm{NIT}) / \mathrm{NIT}-8.8
\end{gathered}
$$

$\mathrm{E}$ represented DPN group, $\mathrm{N}$ represented normal group. PL is the distance between the third toe and heel, TS is the distance between the first and fifth toes. IT is the distance between the second and fourth toes. Generally, a SFI value around 0 indicated normal nerve function, and a value around 100 indicated total dysfunction. SFI was a negative value and a higher
SFI meant the better function of the sciatic nerve. All experiments were repeated by at least two separate investigators and the person who performed the surgeries never participated in the behavioral experiments.

\section{Cell Culture and Treatment}

RSC 96 cells (a rat Schwann cell line) were obtained from ScienCell Research Laboratories. They were cuilured in Dulbecco's modified Eagle Medium (DMEM) containing $5.5 \mathrm{mM}$ D-glucose supplemented with $100 \mathrm{U} \mathrm{ml}^{-1}$ penicillin, $100 \mathrm{mg} \mathrm{ml}^{-1}$ streptomycin and $10 \%$ fetal bovine serum, and incubated in a humidified atmosphere containing $5 \% \mathrm{CO}_{2}$ at $37^{\circ} \mathrm{C}$. After two passages, RSC96 cells were plated at a density of 5000 per well in a 96-well plate for the MTT assay. For protein extraction and apoptosis assay, cells were seeded at a density of $1-5 \times 10^{5} / \mathrm{ml}$ in 6-plate well and permitted to attach and grow for $24 \mathrm{~h}$. The RSC96 cells in each experiment were divided into four groups: (1) the control group; (2) the high glucose (HG) group (30 mM high glucose DMEM); (3) the HG $(30 \mathrm{mM})+$ Free GFs (Each GF was added at a final 50 ng $\mathrm{mL}^{-1}$ ) group; and (4) the HG $(30 \mathrm{mM})+\mathrm{GFs}$ coacervate (the same concentration of GFs) group. All treatments lasted for $24 \mathrm{~h}$.

\section{Western Blot Analysis}

For protein analysis of in vivo samples, the frozen sciatic nerves were homogenized in lysis buffer containing $137 \mathrm{mM} \mathrm{NaCl}, 20 \mathrm{mM}$ Tris- $\mathrm{HCl}$ (pH 7.5), $1 \% \mathrm{NP} 40$ and a protease inhibitor cocktail $\left(10 \mu \mathrm{ml}^{-1}\right.$; GE Healthcare Biosciences, Pittsburgh, PA, USA). The complex was then centrifuged at 12,000 rpm, and the supernatant was obtained for protein assay. For in vitro samples, SCs were lysed in RIPA buffer $(25 \mathrm{mM}$ Tris- $\mathrm{HCl}, 150 \mathrm{mM} \mathrm{NaCl}, 1 \%$ Nonidet P-40, 1\% sodium deoxycholate, and $0.1 \%$ sodium dodecyl sulfate) with protease and phosphatase inhibitors. Protein concentrations were quantified using a BCA Protein Assay Kit (Thermo, Rockford, IL, USA). The equivalent of $80 \mu \mathrm{g}$ of total protein was loaded onto SDS-PAGE and transferred to PVDF membrane (Bio-Rad), and the membrane was blocked with 5\% non fat-milk in TBS with $0.05 \%$ Tween 20 (TBST) for $1.5 \mathrm{~h}$. Primary antibodies were incubated overnight at $4^{\circ} \mathrm{C}$ with the following optimized dilutions: GRP78 (1:1000), ATF-6 (1:1000), XBP-1 (1:300), ATF-4 (1:1000), Caspase-12 (1:1000), CHOP (1:300) and GAPDH (1:10000). The membranes were washed with TBST 3 times and incubated with horseradish peroxidase-conjugated secondary antibodies (1:10000) for $1 \mathrm{~h}$ at room temperature. Signals were visualized using the ChemiDocTM XRS +Imaging System (Bio-Rad). The intensity of immunoreactivity was 
quantified using Java's freely available NIH Image J software. Experiments were repeated three times. GAPDH was used as an internal control.

\section{Immunohistochemistry and histology}

The collected sciatic nerve tissue was post-fixed in cold $4 \%$ paraformaldehyde overnight, and embedded in paraffin. Transverse sections of 5- $\mu \mathrm{m}$ thickness were cut, and the lesion epicenter stained with hematoxylin and eosin (H\&E). To determine the CHOP and Caspase-12 activities, the slides were incubated with $0.3 \% \mathrm{H}_{2} \mathrm{O}_{2}$ in methanol for $30 \mathrm{~min}$, followed by incubating with $10 \%$ normal donkey serum for $1 \mathrm{~h}$ at room temperature in PBS containing $0.1 \%$ Triton X-100. Next, the sections were incubated at $4^{\circ} \mathrm{C}$ overnight with a primary antibody against Caspase-12 (1:1000) and CHOP (1:100). After primary antibody incubation, sections were washed three times for $10 \mathrm{~min}$ with PBS and then incubated with Alexa Fluor 488 (1:1000) or TR-conjugated secondary antibodies for $1 \mathrm{~h}$ at room temperature. Sections were rinsed three times with PBS and incubated with 4,6-diamidino-2-phenylindole (DAPI) for $5 \mathrm{~min}$ and finally washed with PBS and sealed with a coverslip. The images were captured using a Nikon ECLPSE 80i microscope.

\section{Apoptosis Assay}

DNA fragmentation in longitudinal paraffin-cut sections $(5 \mu \mathrm{m})$ of sciatic nerves at the lesion site was detected using the TUNEL apoptosis assay kit (Beyotime Institute of Biotechnology) according to the manufacturer's protocol. Sections were counter-stained with DAPI (blue color), Images were taken at $\times 400$ and the number of TUNEL-positive cells in each section was counted through Image-Pro Plus software. Apoptosis in cultured RSC 96 cells was assessed by FACScan flow cytometer (Becton Dickinson, Franklin Lakes, NJ, USA) as the manual description flow-cytometry using a PI/Annexin V-FITC kit (Invitrogen, Carlsbad, CA, USA). Early and late apoptosis were evaluated by the ratio of fluorescence 2 (for propidium iodide) intensity versus fluorescence 1 (for annexin) intensity plots. The percentage of cells stained by annexin $\mathrm{V}$ alone was recorded as early apoptosis, whereas the percentage of cells stained by both annexin $\mathrm{V}$ and propidium iodide was recorded as late apoptosis.

\section{Statistical Analysis}

The data were expressed as the mean \pm SD. Statistical differences were evaluated using One-way analysis of variance (ANOVA) followed by Tukey's test with GraphPad Prism 5 software (GraphPad Software Inc., La Jolla, CA, USA). For all comparisons, values of $p<0.05$ were considered statistically significant.

\section{Results}

\section{Release of GFs bound to coacervate}

To investigate the amount of GFs that was sequestered into the coacervate, we immersed the GFs- coacervate in saline buffer and measured the release rates of bFGF and NGF by ELISA on Day 1, 4, 7, 14, 21, 28 and 35. As showed in Figure 1, the release rate of NGF is faster than bFGF. At the 1st day, the coacervate released about 1,520 ng NGF, which was more than the bFGF whose release quantity was about $480 \mathrm{ng}$. Afterwards, both of GFs release curve showed nearly linear and sustained trend. Until the day 35, about $66.4 \%$ of bFGF still retained on the coacervate, but NGF is only about $33.1 \%$. Considering the dissociation constant $(\mathrm{Kd})$ of bFGF $(2 \mathrm{nM})$ and NGF $(600 \mathrm{nM})$, it is easily to understand that the binding property of bFGF to heparin-PEAD is more stronger than NGF, leading to different release rate. Because the release rate of incorporated GFs in vivo is also influenced by enzymes such as heparinase or erepsin, one would expect a faster rate of release in vivo. Next, we would systematically evaluate the role of GFs coacervate for DPN restoration in diabetic rats.

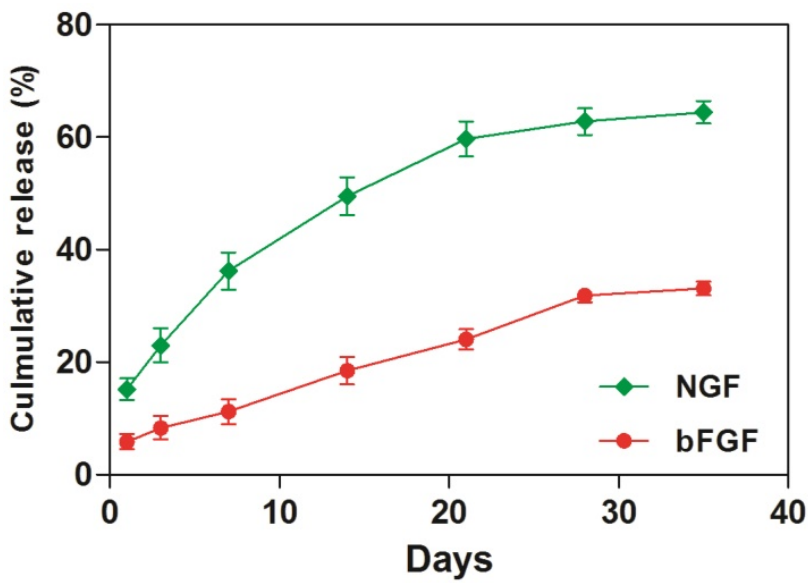

Figure 1. The coacervate in vitro controls the release of bFGF and NGF for 35 days in a steady fashion. Equal amount of bFGF and NGF were combined, then mixed with heparin followed by PEAD to form coacervate. After centrifuged, supernatant was collected. The amount of released bFGF and NGF was measured by ELISA at day 1, 4, 7, 14, 21, 28, and 35, Data were presented as means $\pm S D$ ( $n=3$ per group).

GFs coacervate prevents progression of motor and sensory neural dysfunction with no influence on body weight and blood glucose

We then used the walking track analysis and hot plate test to compare the efficacy of two different treatment approaches: free GFs versus GFs coacervate, as potential intervention therapies for 
DPN. We divided the STZ-induced rats into three groups (STZ-diabetes, free GFs, GFs coacervate) and there was no significant difference among the three groups prior to drug treatments. These rats were then treated and tested, as described, for up to 5 weeks.

Our results showed that the free GFs treatment at Day 7 significantly increased the hindlimb locomotor function and withdrawal latency, even better than the GFs coacervate treated group (Figure $2 \mathrm{~B}$ and $\mathrm{C}$ ). 14 days after treatment, the SFI value and the withdrawal threshold of the free GFs group was similar to that in GFs coacervate group. However, from day 21 on, these two parameters exhibited significant differences among the three diabetic groups; the free GFs group showed a distinct recovery in motor and sensory function compared to the STZ-diabetes group, but inferior to that in GFs coacervate group $(\mathrm{n}=8, \mathrm{p}<0.05)$. This different became even more noticeable at day 28 .

All animals were measured for their blood glucose level and for their body weight at 8 weeks before drug treatment, and at sacrificed time following drug treatment. All STZ-received rats showed significantly higher blood glucose levels than those of control groups but weren't different in themselves. Interestingly, the blood glucose level of
DPN rats remained unchanged after they injected with Free GFs or GFs coacervate (Table 1). The changes in body weight in all groups showed a trend similar to the plasma glucose change. These results suggest that GFs coacervate can effectively promote functional improvement of motor and sensory recovery without affecting plasma glucose levels and body weight in DPN rats.

\section{GFs coacervate ameliorates neurological morphology in DPN rats}

At 30 days after treatment, demyelination and fiber irregularity of sciatic nerve were evaluated by H\&E staining. Axonal atrophy with irregularity of neuropile and unclear boundary in myelin sheaths were seen in sciatic nerves of STZ-diabetes group. In contrast, this change appeared to be mild in free GFs-treated group, mainly with nerve fibers of ordered arrangement and myelin sheaths of extensive regeneration. Yet, the GFs coacervate group had the best regularity of arranged nerve fibers, and the boundary of myelin sheaths evidently clear (Figure 3). These results indicated that GFs coacervate was able to counteract against the morphology changes associated with diabetic neuropathy of the sciatic nerve.

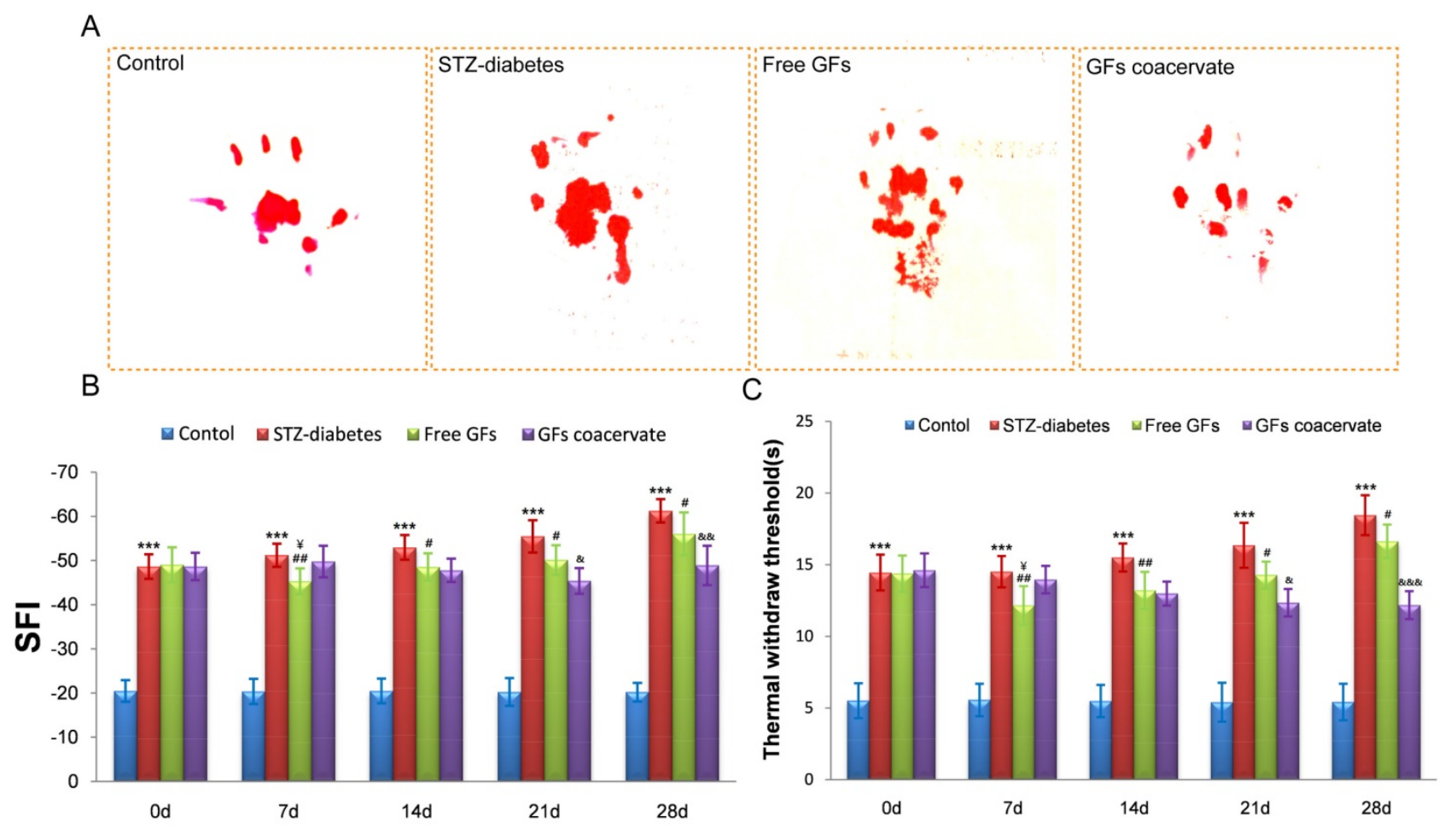

Figure 2. The recovery of motor and sensory function was evaluated by walking track analysis and hot plate test. (A) Photographs of the rats' footprints in each group at 30 days after treatment. (B), (C) Statistical analysis of sciatic function index (SFI) value and thermal withdraw threshold. ${ }^{* * *} \mathrm{P}<0.001$ versus control group, $\# \mathrm{P}<0.05$, $\# P<0.01$ versus STZ-diabetes group, $\& P<0.05$, \&\& $P<0.01$, \&\&\& $P<0.001$ versus the free GFs group, $¥ \mathrm{P}<0.05$ versus the $G F s$ coacervate group. Data are the mean values $\pm \mathrm{SD}, \mathrm{n}=8$. 


\section{Control \\ STZ-diabetes \\ Free GFs \\ GFs coacervate}

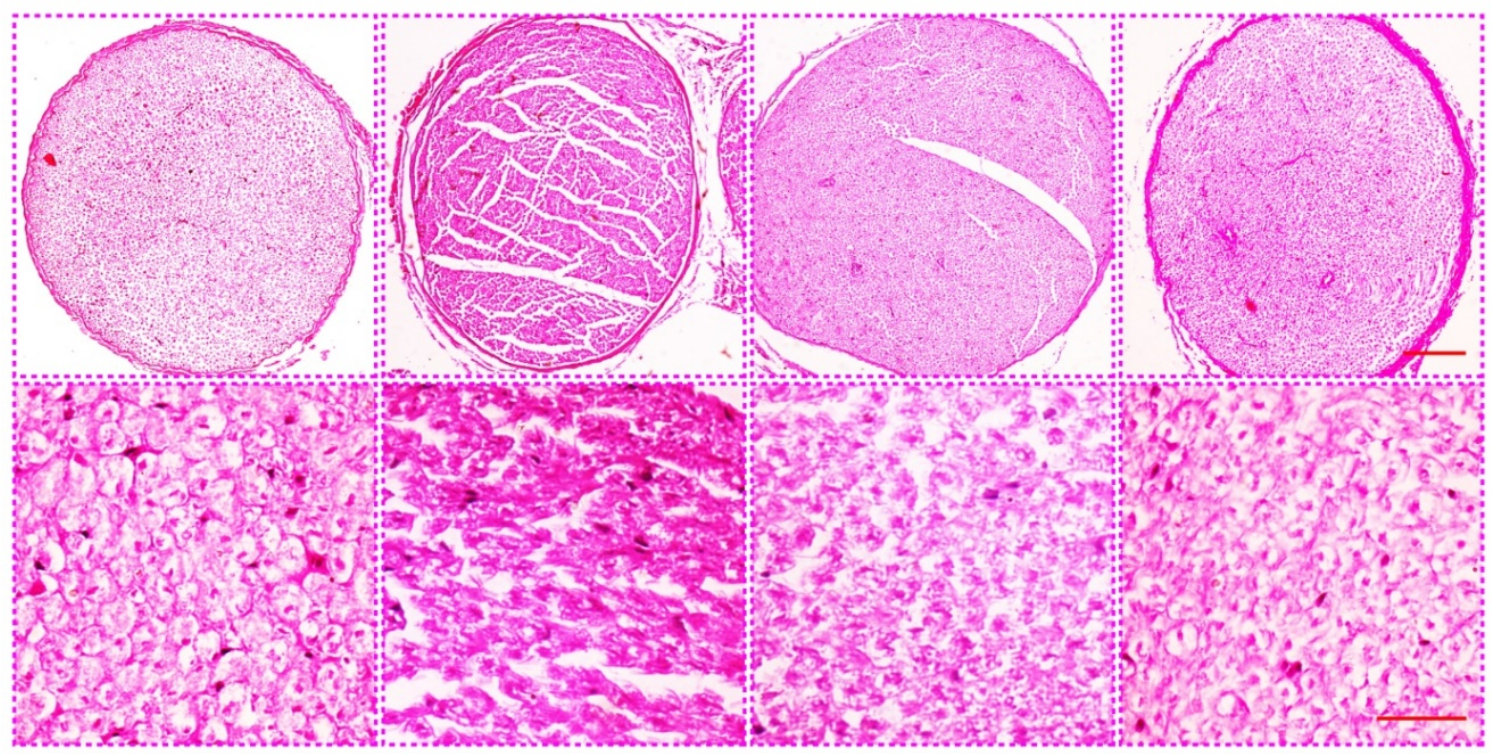

Figure 3. H\&E staining results of cross-sectional tissue slices of control, STZ-diabetes, free GFs, and GFs coacervate group respectively. The above magnification was $10 \times$ and the below magnification was $40 \times$.

\section{GFs coacervate decreases cells apoptosis in the sciatic nerve with diabetic neuropathy}

As a measure of DNA fragmentation, TUNEL staining with sections of sciatic nerves were obtained in each group at day 30 after treatment. As shown in Figure 4A, the number of TUNEL-positive cells in diabetic sciatic nerve without drug administration was significantly increased, when compared with those in the control animals $(\mathrm{P}<0.001)$. On the contrary, the number of increased apoptotic cell in diabetic sciatic nerve considerably reduced when treated with free GFs or GFs coacervate. Interestingly, the reverse effect of the GFs coacervate group was better than the free GFs group, nearly reached the same as control tissue $(\mathrm{P}<0.05$, Fig. $4 \mathrm{~B})$. These results demonstrated that GFs coacervate exhibited significantly protective effects on sciatic nerve of DPN rats with fewer apoptotic cells.

Table 1. Body weights and blood glucose concentrations in nondiabetic and STZ diabetic rats treating with free GFs or GFs coacervate.

\begin{tabular}{lllll}
\hline Group & \multicolumn{2}{l}{ Body weight $(\mathrm{g})$} & \multicolumn{2}{l}{ Blood glucose $(\mathrm{mmol} / \mathrm{L})$} \\
\cline { 2 - 5 } & 0 day & 30 days & 0 day & 30 days \\
\hline Control & $422 \pm 15$ & $437 \pm 13$ & $6.5 \pm 0.5$ & $6.5 \pm 0.6$ \\
STZ-diabetes & $230 \pm 11^{* *}$ & $219 \pm 13^{* *}$ & $20.9 \pm 2.1^{* * *}$ & $22.6 \pm 2.3^{* * *}$ \\
Free GFs & $231 \pm 14$ & $227 \pm 11$ & $21.0 \pm 2.3$ & $20.8 \pm 2.8$ \\
GFs coacervate & $227 \pm 18$ & $229 \pm 15$ & $20.2 \pm 2.0$ & $20.3 \pm 2.2$ \\
\hline
\end{tabular}

Data are expressed as mean $\pm S D, n=8$ per group. ${ }^{* *} \mathrm{P}<0.01,{ }^{* * *} \mathrm{P}<0.001$ versus the control group.

\section{GFs coacervate inhibits excessive ERS in the DPN rats}

ERS activation is related to DPN [5, 26]. To test whether or not ERS is involved in neuroprotective role of GFs on DPN, we firstly investigated the expression level of ERS in neural tissues of STZ diabetic rats after treating with free GFs or GFs coacervate. In samples from rats with a 12-week post diabetes induction, Western blot results revealed a clear upregulation of GRP-78, ATF-6, XBP-1, ATF-4, CHOP and Caspase-12, all well-recognized markers for ERS, suggesting that ERS is involved in DPN development and progression.

With free GFs treatment, the rise in these stress markers expression was decreased. In addition, the levels of these stress markers in GFs coacervate group were significantly lower than those in free GFs group, this trend also corresponded with the statistical analysis (Figure 5A, B). To further confirm the role of GFs coacervate on ERS, CHOP and Caspase-12 expression in sciatic nerve sections of DPN rats were detected by immunofluorecent staining analysis. In the line of Western blot changes, the CHOP and Caspase-12 positive red or green signals in the free GFs group were significantly less than the control group, but more than GFs coacervate (Figure 5C), which suggests that GFs coacervate has a better capacity of inhibiting excessive ERS in sciatic nerves of DPN rats than free GFs groups. Taken together, these results strongly suggest that GFs coacervate is more efficacious in attenuating ERS response after DPN. 


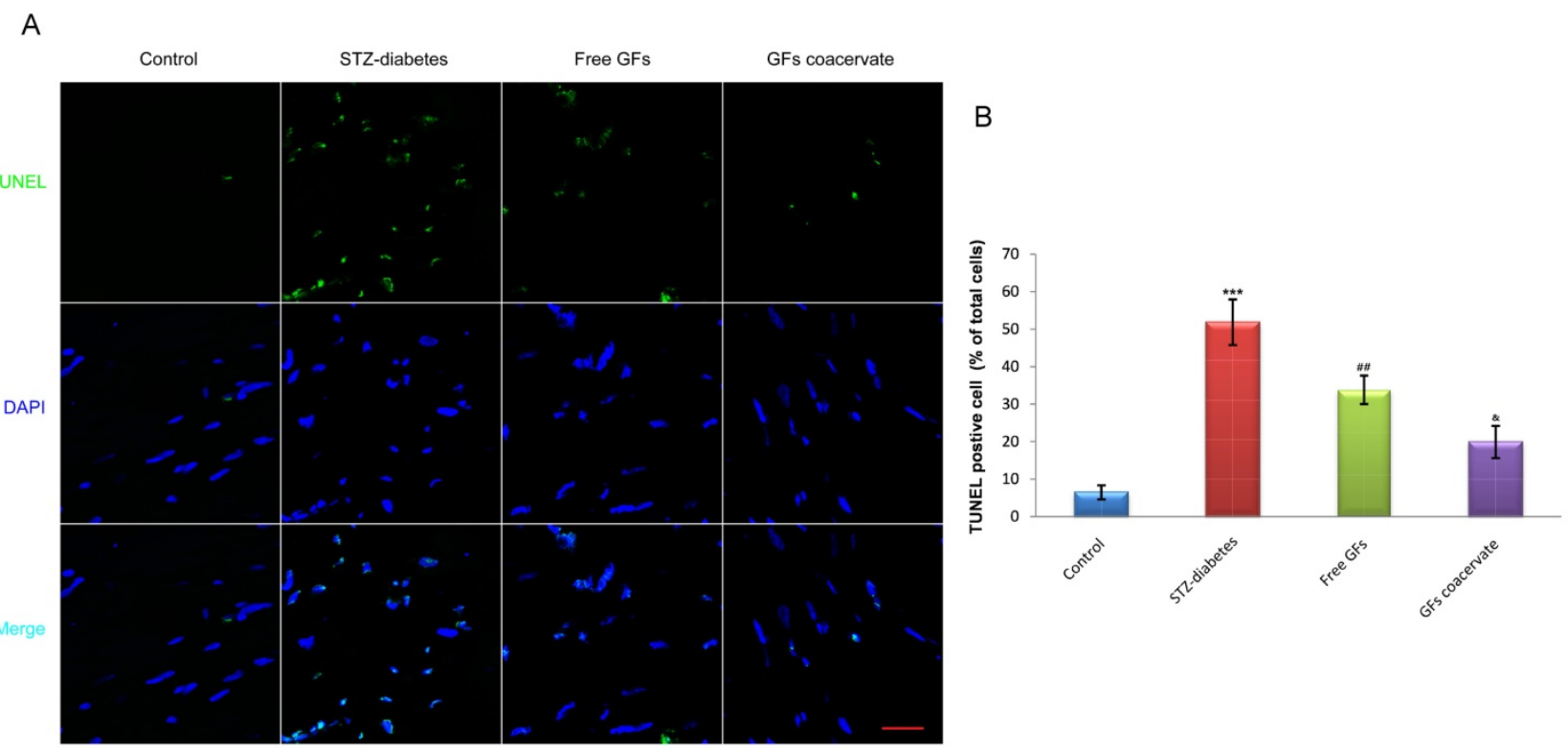

Figure 4. GFs coacervate decreases the level of apoptosis in sciatic nerve lesions. (A) Representative micrographs showing immunofluorescence with TUNEL (green). Nuclei are labeled with DAPI (blue), Scale bars $=50 \mu \mathrm{m}$. (B) The percentage of TUNEL-positive cells in the sciatic nerve microsection of four groups. ${ }^{*} \mathrm{P}<$ 0.001 versus the control group, $P<0.01$ versus the STZ-diabetes group, \& $P<0.05$ versus the free GFs group. Results are mean \pm SD of three independent experiments.

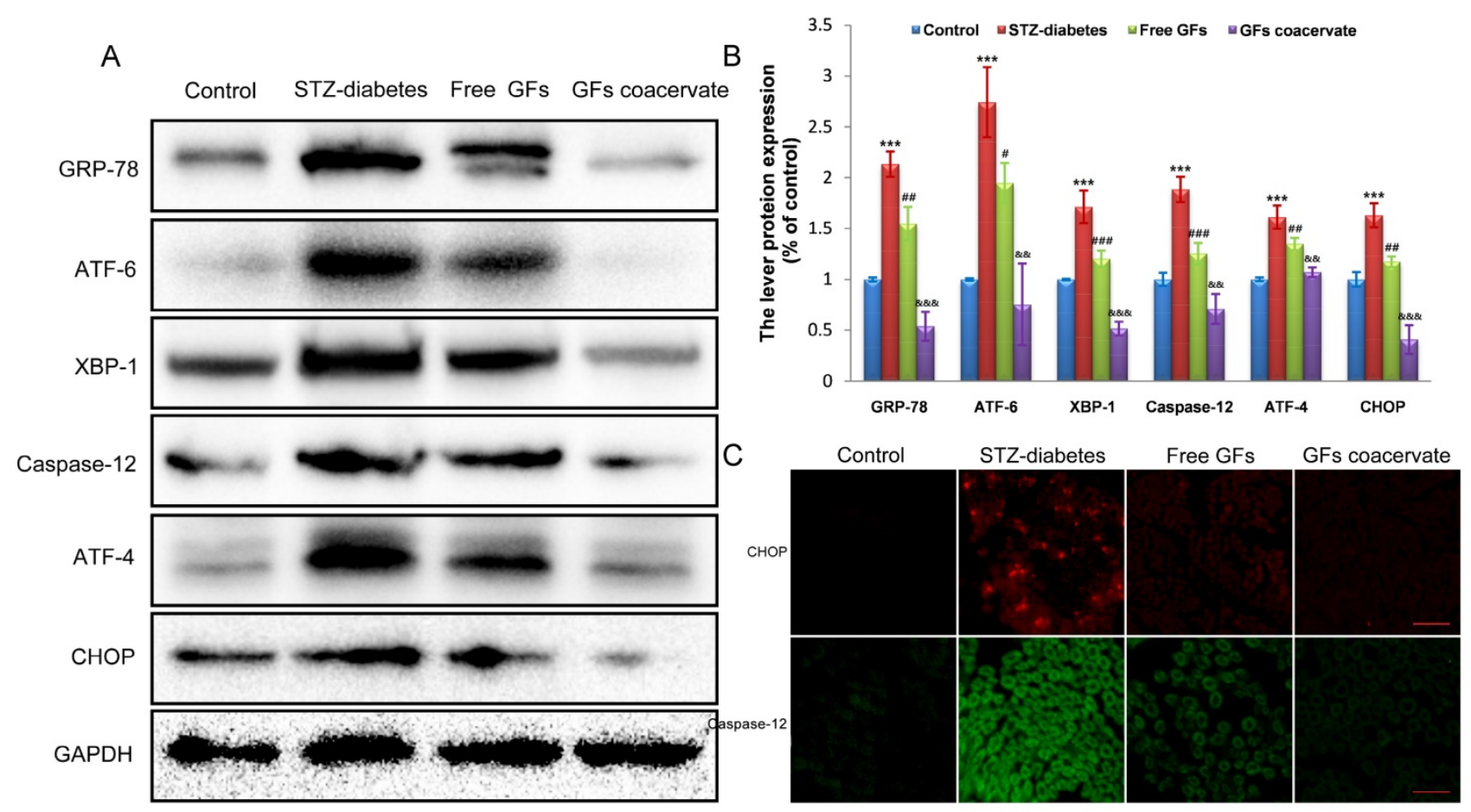

Figure 5. GFs coacervate administration inhibits excessive ERS in the DPN rats. (A) Sciatic nerve tissues samples were analyzed with Western Blotting for the expression of GRP-78, ATF-6, XBP-1, ATF-4, CHOP and Csapase-12. GAPDH was used for band density normalization. (B) The optical density analysis of GRP-78, ATF-6, XBP-1, ATF-4, CHOP and Csapase-12. $\mathrm{P}<0.001$ versus the control group, $\# \mathrm{P}<0.05, \ldots \mathrm{P}<0.01$, $\mathrm{P} \mathrm{P}<0.001$ versus the STZ-diabetes group, \&\& $\mathrm{P}<0.01$, z\&\& $P<0.001$ versus the free GFs group. Mean values $\pm S D, n=8$. (C) Immunofluorescent staining of $C H O P$ and caspase- 12 in each group. Scale bars $=50 \mu m$.

\section{GFs coacervate reduces the HG-induced apoptosis of RSC96 Cells in vitro}

To further ensure the remarkable protective function of GFs coacervate, we tested its impact on hyperglycaemia-induced apoptosis in a cellular model, RSC96 cells. The cells were cultured in $30 \mathrm{mM}$ HG with or without GFs coacervate treatment. Based on our results of MTT assays, HG-induced apoptosis was markedly decreased after treating with GFs 
coacervate (Fig. 6 A), and it was better than only free GFs treatment. We then used fluorescence activated cell sorting analysis to distinguish and measure viable, early apoptotic, late apoptotic or necrotic cells to further demonstrate the antiapoptotic effect of GFs coacervate on RSC96 cells. Our results showed that both GFs coacervate and free GFs clearly inhibited the apoptotic cells as compared to HG group (Figure 6 B, C), and GFs coacervate was more effective than the free GFs. Collectively, these data indicate that GFs coacervate is very effective in inhibiting HG-induced apoptosis.

\section{GFs coacervate protects HG-cultured RSC96 cells by inhibiting excessive ERS in vitro}

To determine whether suppression of chronic ERS is involved in GFs coacervate mediated antiapoptotic effect, we detected the levels of the ERS response proteins including GRP-78, ATF-6, XBP-1, Caspase-12, ATF-4 and CHOP in RSC96 cells by Western blot methods. Consistent with the data in vivo, HG treatment induced significant increases in the levels of GRP-78, ATF-6, XBP-1, Caspase-12, ATF-4 and CHOP expression. Although treatment with free GFs reversed the up-regulation of these protein levels, GFs coacervate treatment was more effective in these measures (Figure 7A, B). Taken together, these results suggested that GFs coacervate treatment reduced ERS, resulting in the suppression of SCs apoptotic under HG condition.

\section{Discussion}

DPN is a chronic complication that seriously affects the quality of life of diabetes person and occurs in $60-70 \%$ of patients [27] with symptoms including spontaneous pain, hyperalgesia, and diminished sensation [28]. Currently, the only effective treatment to prevent or retard the development of DPN and alleviate symptoms is glucose control and pain management [4]. However, its role in recovery of the structure and function for nervous system in established DPN is controversial. Unfortunately, present drug therapy for DPN are mainly including aldose reductase inhibitor (ARI), B vitamins, essential fatty acids $\gamma$-linolenic acid and the antioxidant a-lipoic acid. Moreover, many of them just provide symptomatic treatment; even some of them show adverse side effects [29]. For example, Tolrestate is a kind of ARI compound that can action on DPN, but showed disappointed clinical efficacy [30]. Thus, seeking for new therapeutic strategies on DPN becomes necessary.

A

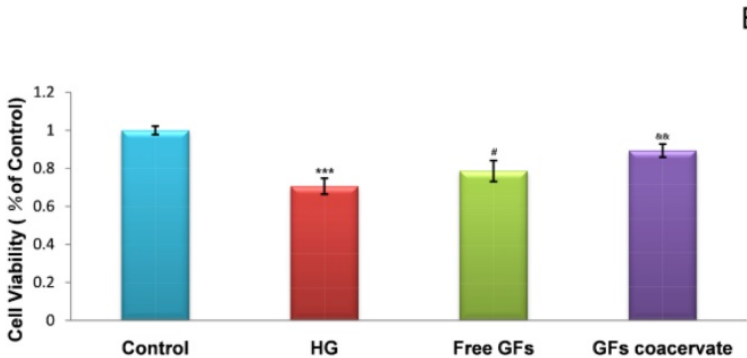

C

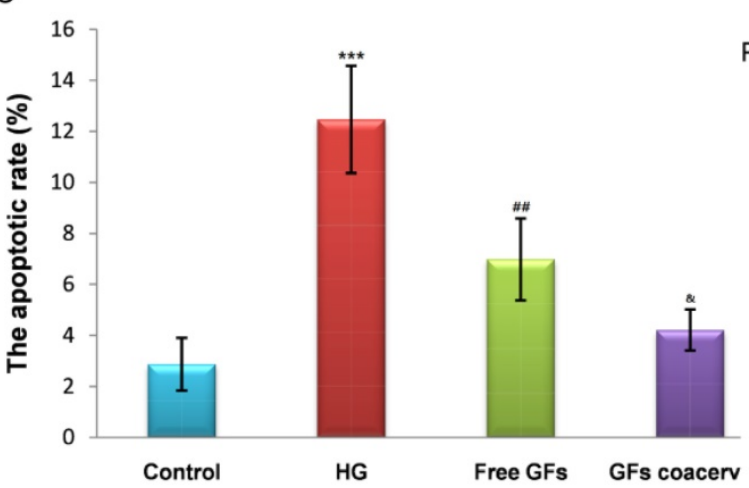

B
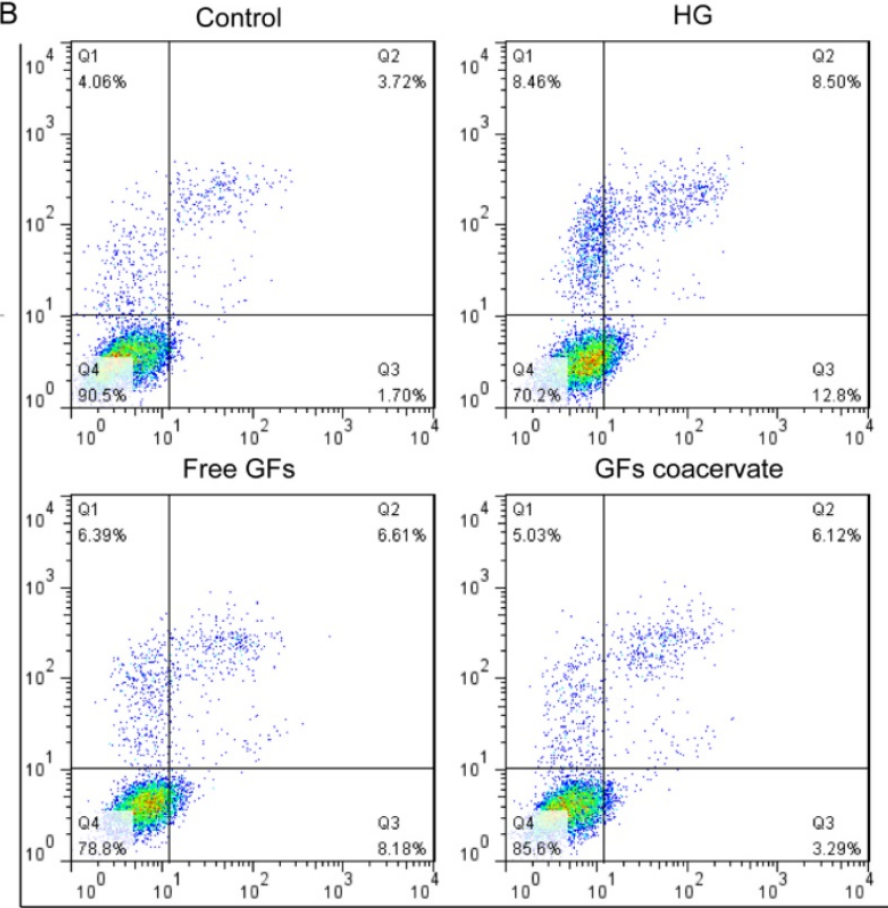

Annexin V-FITC

Figure 6. GFs coacervate suppresses HG-induced apoptosis in SCs. (A) SCs were treated with free GFs or GFs coacervate for 24 h under HG conditions and then cell viability was assessed by MTT assay. (B) Flow cytometry result of PI/Annexin V-FITC staining for cell apoptosis analysis. (C) Quantitative analysis of the early apoptotic rates from three separate experiments. ${ }^{* * *} P<0.001$ versus the control group, $\#<0.05$, \# $P<0.01$ versus the $H G$ group, \& $P<0.05$, \&\& $P<0.01$ versus the free GFs group. 


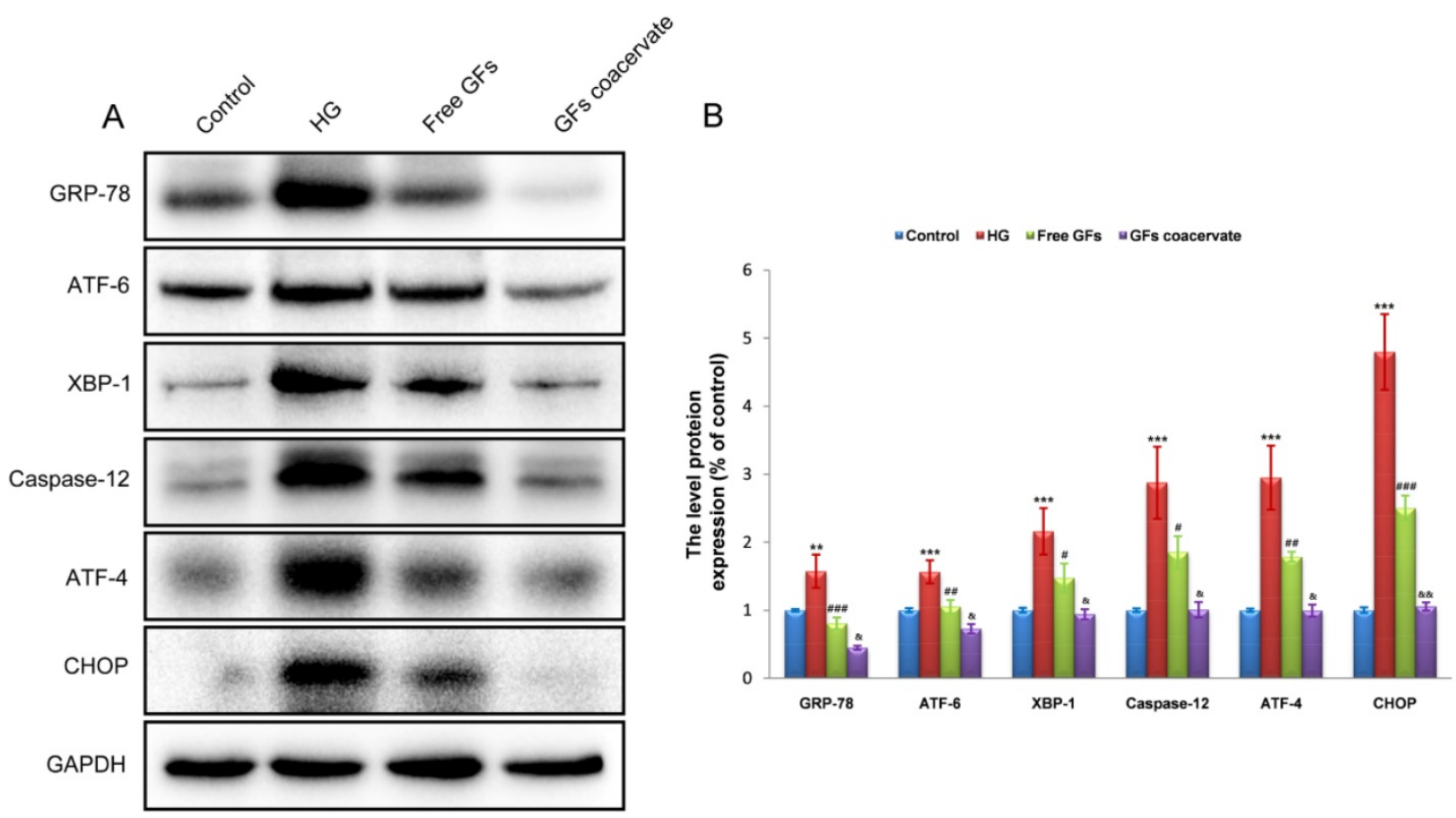

Figure 7. GFs coacervate attenuates HG-induced ERS in SCs. (A) Representative western blot results of ERS markers: GRP-78, ATF-6, XBP-1, ATF-4, CHOP and

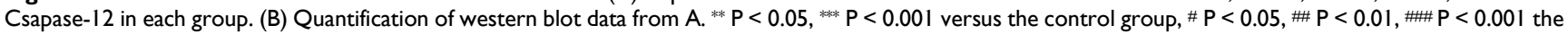
HG group, \& $P<0.05$, \&\& $P<0.01$ versus the free GFs group. All data are represent as mean values $\pm S D, n=3$.

NTFs, including bFGF and NGF, provide trophic and tropic support that are vital for PNS development and function. NGF regulates intraneural homeostasis during development by providing neurotrophic and regulating intracellular pathways to promote neuronal sprouting [31]. bFGF is a mitogenic cationic polypeptide that can facilitate neurite extension and stimulate SCs proliferation, as well as inducing angiogenesis [32]. Both exogenous NGF and bFGF have been shown to have protective effects in rodents with DPN $[14,33]$. Furthermore, a deficiency of those two GFs may cause neuron susceptible to injury and death $[34,35]$. A number of studies are mainly focus on administrating only one kind of those two cytokines to investigate the therapeutic effectiveness. Since they have the synergistic effect on DPN, why not combine them together? This combinatorial therapy on NGF and bFGF in our work is distinguished from all the moment research. However, as the kind of protein component, free GFs have a short circulating half-life and degrade easily in body fluids [20, 21]. So it is difficult for GFs to be retained at lesion sites. Seeking for a controlled delivery system that can preserve their processibility and biocompatibility would be particularly attractive for biomedical applications. Thus, we used a coacervate protein delivery platform that consists of native heparin and a novel polycation, poly (ethylene argininylaspartate diglyceride) (PEAD), to deliver GFs. As demonstrated on Figure 1, this
[PEAD:heparin] coacervate not only combined bFGF and NGF in a massive loading capability but also persistently and steadily controlled their release for at least 35 days. Our study revealed that only single administration of the GFs coacervate was sufficient for the persistent reparation of diabetic neuropathy. Moreover, GFs delivered by the coacervate promotes better nerve recovery than dual therapy with free GFs.

In DPN model of diabetic rats, we firstly assessed functional recovery of sensory and motor systems after treating with GFs using walking track analysis and hot plate test. Free bFGF and NGF treatment induced a transient improvement in these behavioural tests, however, sustained improvement over four weeks was observed with the combined bFGF and NGF coacervate treatment relatived to the untreated diabetic group. Additionally, GFs coacervate was also found to improve sciatic nerve morphology including ameliorating unclear boundary in myelin sheaths, and reducing the loss of nerve fibers, as well as significantly reducing SCs apoptosis (Figure 3 and 4). Interestingly, the body weight and blood glucose level in DPN rats remained unchanged after treating with free GFs or GFs coacervate, indicating that the neuroprotective effect of GFs or GFs coacervate on DPN doesn't depend on the regulation of glucose level and body weight.

The pathophysiological mechanisms of DPN are multifactorial, which includes enhanced polyol pathway activities, increased advanced glycation end 
products, altered hexosamine pathways, and producted massive free radical. ERS, as a momentous mechanism for metabolic diseases, have been reported extensively [36, 37], especially for DPN. Lupachyk et al found that proteins implicated in ERS were overexpressed in spinal cord and sciatic nerve of STZ-induced diabetic rodents. And administration of ERS inhibitors could suppress the levels of GRP78, GRP94, ERO1 $\alpha$ and CHOP expression, accompanied by the recovery of neurological function [6]. They also observed that sciatic nerve dysfunction and glucose intolerance were alleviated in high-fat diet mice and Zucker ( $\mathrm{fa} / \mathrm{fa}$ ) rats when treated with trimethylamine oxide, a selective inhibitor of eukaryotic initiation factor-2alpha (eiF2a) [26]. In the present study, the change levels of ERS response proteins including GRP-78, ATF-6, XBP-1, ATF-4, CHOP and Csapase-12 were tested to confirm whether ERS is involved in the beneficial role of GFs coacervate on DPN [5]. As shown in Figure 5, GFs coacervate treatment significantly reduced upregulation of ERS proteins in the STZ-diabetic rats more than those in treated with free GFs. This result was also confirmed by immunofluorescence analysis. Taken together, these data suggest that GFs coacervate may be related to the inhibition of ERS, consequently suppressing SCs apoptosis.

As spongiocyte in the PNS, SCs exert a pivotal role in supporting axon outgrowth and regeneration, and forming myelin sheath to help repair damaged nerves [38]. Under HG condition, the SCs' injury was earlier than ongoing myelinic denaturation and axonal atrophy [39-41]. Because of the similar characteristics on primary SCs, the cultured rat Schwann cell line-RSC 96 cells in $30 \mathrm{mM}$ high-glucose (HG) medium were able to mimic metabolic changes of diabetic patients with DPN. As shown in Figure 6 and 7, SCs in HG induced a significantly high level of ERS response proteins and caused a higher apoptotic rate compared to the control group. This apoptotic activity was suppressed by both free GFs or GFs coacervate treatment, however, the inhibitory effect of GFs coacervate was significantly greater than that of free GFs. Indeed, the current research has verified that GFs coacervate plays a vital role in anti-apoptosis by inhibiting excessive ERS activation of SCs under the hyperglycemic condition.

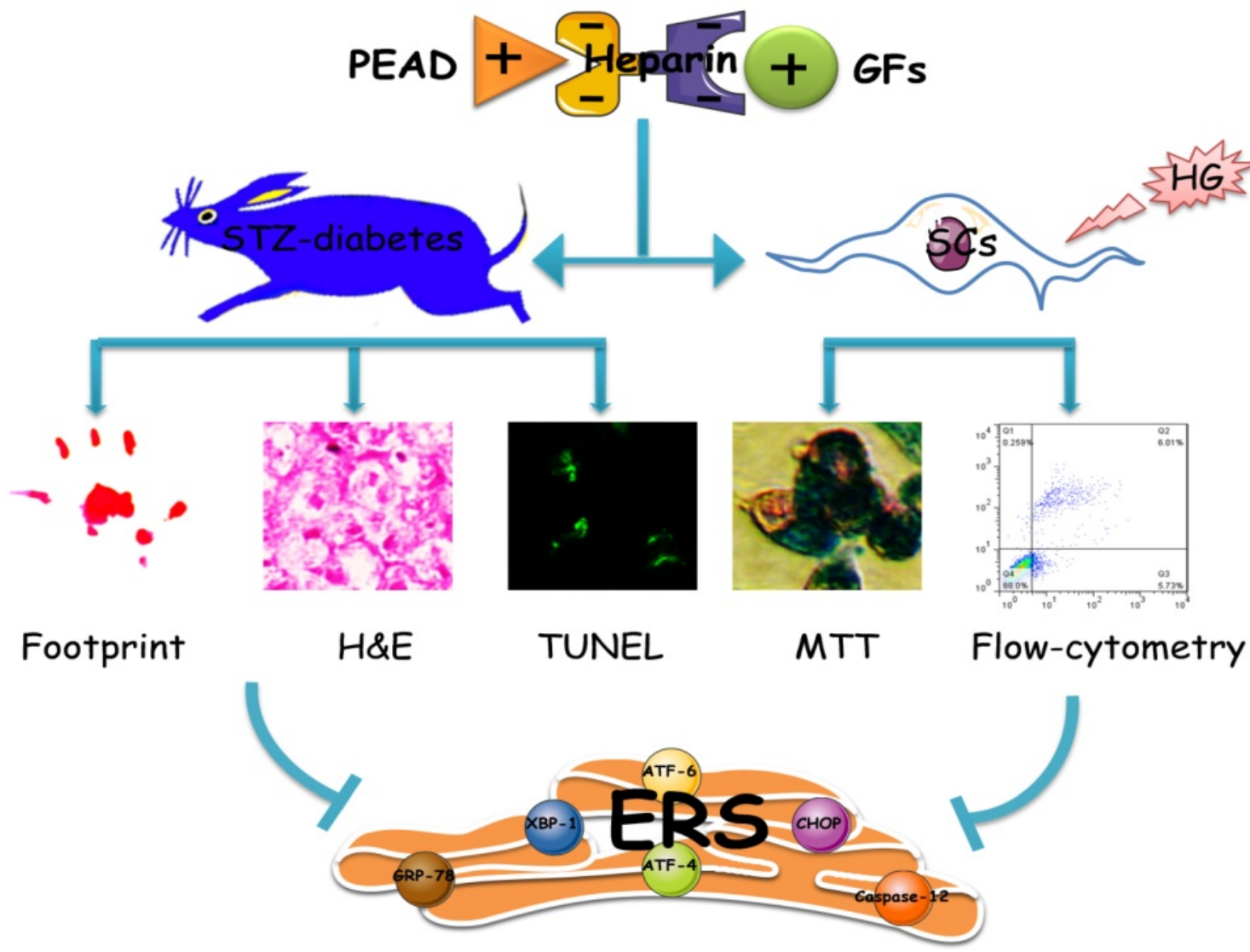

Figure 8. Illustrating the therapeutic effect of NGF+bFGF : heparin : PEAD coacervate on DPN-rats and HG-SCs and its mechanism. For the STZ-induced diabetic rats, GFs coacervate mediated nerve regeneration and functional recovery by improved motor and sensory with more ordered nerve fibers and regenerated myelin sheath as well as suppressed apoptosis of SCs. Furthermore, the molecular mechanism of the GFs coacervate protective effect is involved in inhibiting excessive ERS-induced SCs apoptosis. 
In conclusion, as shown in Figure 8, this heparin-based coacervate had been developed for co-delivery of bFGF and NGF, which shown its property to combine GFs with high load efficiency and controlled their release at least 35 days. GFs coacervate recovered the function and structure of sciatic nerve by improved motor and sensory with more ordered nerve fibers and regenerated myelin sheath as well as suppressed apoptosis of SCs in DPN rats. Moreover, the inhibition of ERS-induced apoptosis is the molecular mechanism of the GFs coacervate protective effect on DPN, highlighting protective role of GFs coacervate in ERS and apoptosis of Schwann cell may be a useful therapeutic strategy for DPN. Further experimental and clinical studies will focus on evaluating its efficacy and long-term safety, which may promote the application of GFs coacervate as a treatment modality for diabetic neuropathy.

\section{Abbreviations}

GFs: growth factors; GF: growth factor; NTFs: neurotrophic factors; bFGF: basic fibroblast growth factor; NGF: nerve growth factor; DPN: diabetic peripheral neuropathy; PNS: peripheral nervous system; SCs: Schwann cells; ER: endoplasmic reticulum; ERS: endocytoplasmic reticulum stress; UPR: unfolding protein response; PEAD: poly (ethylene arginyl aspartate diglyceride); STZ: streptozotocin; HG: high glucose; eiF2a: eukaryotic initiation factor-2alpha; ELISA: enzyme linked immunosorbent assay; DMEM: Dulbecco's modified Eagle's medium; FBS: fetal bovine serum; PBS: phosphate-buffered saline; TWRL: Thermal withdrawal reflex; SFI: the sciatic function index; DAPI: 4,6-diamidino-2-phenylindole; H\&E: hematoxylin and eosin; ANOVA: analysis of variance.

\section{Acknowledgements}

This study was partially supported by a research grant from the National Natural Science Funding of China (81372112), Zhejiang Provincial Program for the Cultivation of High-level Innovative Health Talents (to J.X.). J.W. would like to thank the financial support from Zhejiang Provincial Natural Science Foundation of China (LQ15E030003), Wenzhou Municipal Science \& Technology Bureau of China (Y20140574).

\section{Competing Interests}

The authors have declared that no competing interest exists.

\section{References}

1. Sinnreich M, Taylor BV, Dyck PJ. Diabetic neuropathies. Classification, clinical features, and pathophysiological basis. The neurologist. 2005; 11: 63-79.
2. Singh R, Kishore L, Kaur N. Diabetic peripheral neuropathy: current perspective and future directions. Pharmacological research : the official journal of the Italian Pharmacological Society. 2014; 80: 21-35.

3. Tesfaye S, Boulton AJ, Dyck PJ, Freeman R, Horowitz M, Kempler P, et al. Diabetic neuropathies: update on definitions, diagnostic criteria, estimation of severity, and treatments. Diabetes care. 2010; 33: 2285-93.

4. Callaghan BC, Cheng HT, Stables CL, Smith AL, Feldman EL. Diabetic neuropathy: clinical manifestations and current treatments. The Lancet Neurology. 2012; 11: 521-34.

5. O'Brien PD, Hinder LM, Sakowski SA, Feldman EL. ER stress in diabetic peripheral neuropathy: A new therapeutic target. Antioxidants \& redox signaling. 2014; 21: 621-33.

6. Lupachyk S, Watcho P, Stavniichuk R, Shevalye H, Obrosova IG. Endoplasmic reticulum stress plays a key role in the pathogenesis of diabetic peripheral neuropathy. Diabetes. 2013; 62: 944-52.

7. Hotamisligil GS. Endoplasmic reticulum stress and the inflammatory basis of metabolic disease. Cell. 2010; 140: 900-17.

8. Eizirik DL, Cardozo AK, Cnop M. The role for endoplasmic reticulum stress in diabetes mellitus. Endocrine reviews. 2008; 29: 42-61.

9. Hotamisligil GS. Endoplasmic reticulum stress and atherosclerosis. Nature medicine. 2010; 16: 396-9.

10. Zhang HY, Wang ZG, Lu XH, Kong XX, Wu FZ, Lin L, et al. Endoplasmic reticulum stress: relevance and therapeutics in central nervous system diseases. Molecular neurobiology. 2015; 51: 1343-52.

11. Andrades JA, Wu LT, Hall FL, Nimni ME, Becerra J. Engineering, expression, and renaturation of a collagen-targeted human bFGF fusion protein. Growth Factors. 2001; 18: 261-75.

12. Lane JT. The role of retinoids in the induction of nerve growth factor: a potential treatment for diabetic neuropathy. Translational research : the journal of laboratory and clinical medicine. 2014; 164: 193-5.

13. Hellweg R, Raivich G, Hartung HD, Hock C, Kreutzberg GW. Axonal transport of endogenous nerve growth factor (NGF) and NGF receptor in experimental diabetic neuropathy. Experimental neurology. 1994; 130: 24-30.

14. Nakae M, Kamiya H, Naruse K, Horio N, Ito Y, Mizubayashi R, et al. Effects of basic fibroblast growth factor on experimental diabetic neuropathy in rats. Diabetes. 2006; 55: 1470-7.

15. Tomlinson DR, Fernyhough P, Diemel LT. Role of neurotrophins in diabetic neuropathy and treatment with nerve growth factors. Diabetes. 1997; $46 \mathrm{Suppl}$ 2: S43-9.

16. Chen SQ, Cai Q, Shen YY, Cai XY, Lei HY. Combined use of NGF/BDNF/bFGF promotes proliferation and differentiation of neural stem cells in vitro. International journal of developmental neuroscience : the official journal of the International Society for Developmental Neuroscience. 2014; 38 : 74-8.

17. Wu J, Ye J, Zhu J, Xiao Z, He C, Shi H, et al. Heparin-Based Coacervate of FGF2 Improves Dermal Regeneration by Asserting a Synergistic Role with Cell Proliferation and Endogenous Facilitated VEGF for Cutaneous Wound Healing. Biomacromolecules. 2016; 17: 2168-77.

18. Chen WC, Lee BG, Park DW, Kim K, Chu H, Huard J, et al. Controlled dual delivery of fibroblast growth factor- 2 and Interleukin- 10 by heparin-based coacervate synergistically enhances ischemic heart repair. Biomaterials. 2015; 72: 138-51.

19. Awada HK, Johnson NR, Wang Y. Dual delivery of vascular endothelial growth factor and hepatocyte growth factor coacervate displays strong angiogenic effects. Macromolecular bioscience. 2014; 14: 679-86.

20. Poduslo JF, Curran GL. Permeability at the blood-brain and blood-nerve barriers of the neurotrophic factors: NGF, CNTF, NT-3, BDNF. Brain research Molecular brain research. 1996; 36: 280-6.

21. Yuge T, Furukawa A, Nakamura K, Nagashima Y, Shinozaki K, Nakamura T, et al. Metabolism of the intravenously administered recombinant human basic fibroblast growth factor, trafermin, in liver and kidney: degradation implicated in its selective localization to the fenestrated type microvasculatures. Biological \& pharmaceutical bulletin. 1997; 20: 786-93.

22. Chu H, Johnson NR, Mason NS, Wang Y. A [polycation:heparin] complex releases growth factors with enhanced bioactivity. Journal of controlled release : official journal of the Controlled Release Society. 2011; 150: 157-63.

23. Coppey LJ, Gellett JS, Davidson EP, Dunlap JA, Lund DD, Yorek MA. Effect of antioxidant treatment of streptozotocin-induced diabetic rats on endoneurial blood flow, motor nerve conduction velocity, and vascular reactivity of epineurial arterioles of the sciatic nerve. Diabetes. 2001; 50: 1927-37.

24. Schmeichel AM, Schmelzer JD, Low PA. Oxidative injury and apoptosis of dorsal root ganglion neurons in chronic experimental diabetic neuropathy. Diabetes. 2003; 52: 165-71.

25. Bain JR, Mackinnon SE, Hunter DA. Functional evaluation of complete sciatic, peroneal, and posterior tibial nerve lesions in the rat. Plastic and reconstructive surgery. 1989: 83: 129-38.

26. Lupachyk S, Watcho P, Obrosov AA, Stavniichuk R, Obrosova IG. Endoplasmic reticulum stress contributes to prediabetic peripheral neuropathy. Experimental neurology. 2013; 247: 342-8.

27. Deli G, Bosnyak E, Pusch G, Komoly S, Feher G. Diabetic neuropathies: diagnosis and management. Neuroendocrinology. 2013; 98: 267-80.

28. Vinik AI, Park TS, Stansberry KB, Pittenger GL. Diabetic neuropathies. Diabetologia. 2000; 43: 957-73. 
29. Bosi E, Conti M, Vermigli C, Cazzetta G, Peretti E, Cordoni MC, et al. Effectiveness of frequency-modulated electromagnetic neural stimulation in the treatment of painful diabetic neuropathy. Diabetologia. 2005; 48: 817-23.

30. Pfeifer MA, Schumer MP, Gelber DA. Aldose reductase inhibitors: the end of an era or the need for different trial designs? Diabetes. 1997; 46 Suppl 2: S82-9.

31. Skaper SD. The neurotrophin family of neurotrophic factors: an overview. Methods Mol Biol. 2012; 846: 1-12.

32. Fujimoto E, Mizoguchi A, Hanada K, Yajima M, Ide C. Basic fibroblast growth factor promotes extension of regenerating axons of peripheral nerve. In vivo experiments using a Schwann cell basal lamina tube model. Journal of neurocytology. 1997; 26: 511-28.

33. Aloe L, Rocco ML, Bianchi P, Manni L. Nerve growth factor: from the early discoveries to the potential clinical use. Journal of translational medicine. 2012; 10: 239.

34. Hellweg R, Hartung HD. Endogenous levels of nerve growth factor (NGF) are altered in experimental diabetes mellitus: a possible role for NGF in the pathogenesis of diabetic neuropathy. Journal of neuroscience research. 1990; 26: 258-67.

35. Dobrowsky RT, Rouen S, Yu C. Altered neurotrophism in diabetic neuropathy: spelunking the caves of peripheral nerve. The Journal of pharmacology and experimental therapeutics. 2005; 313: 485-91.

36. Ozcan U, Cao Q, Yilmaz E, Lee AH, Iwakoshi NN, Ozdelen E, et al. Endoplasmic reticulum stress links obesity, insulin action, and type 2 diabetes. Science. 2004; 306: 457-61.

37. Xu C, Bailly-Maitre B, Reed JC. Endoplasmic reticulum stress: cell life and death decisions. The Journal of clinical investigation. 2005; 115: 2656-64.

38. Bhatheja K, Field J. Schwann cells: origins and role in axonal maintenance and regeneration. The international journal of biochemistry \& cell biology. 2006; 38: 1995-9.

39. Eckersley L. Role of the Schwann cell in diabetic neuropathy. International review of neurobiology. 2002; 50: 293-321.

40. Gumy LF, Bampton ET, Tolkovsky AM. Hyperglycaemia inhibits Schwann cell proliferation and migration and restricts regeneration of axons and Schwann cells from adult murine DRG. Molecular and cellular neurosciences. 2008; 37: 298-311.

41. Bestetti G, Rossi GL, Zemp C. Changes in peripheral nerves of rats four months after induction of streptozotocin diabetes. A qualitative and quantitative study. Acta neuropathologica. 1981; 54: 129-34. 